\title{
GAS-GRAIN MODELING OF ISOCYANIC ACID (HNCO), CYANIC ACID (HOCN), FULMINIC ACID (HCNO), AND ISOFULMINIC ACID (HONC) IN ASSORTED INTERSTELLAR ENVIRONMENTS
}

\author{
Donghui Quan ${ }^{1}$, Eric Herbst ${ }^{2}$, Yoshihiro Osamura ${ }^{3}$, and Evelyne RouefF ${ }^{4}$ \\ ${ }^{1}$ Chemical Physics Program, The Ohio State University, Columbus, OH 43210, USA \\ ${ }^{2}$ Departments of Physics, Astronomy, and Chemistry, The Ohio State University, Columbus, OH 43210, USA \\ ${ }^{3}$ Kanagawa Institute of Technology, Atsugi 243-0292, Japan \\ ${ }^{4}$ Observatoire de Paris-Meudon, LUTH UMR 8102, du CNRS and Université Denis Diderot 5 Place Jules Janssen, F-92195 Meudon Cedex, France \\ Received 2010 September 6; accepted 2010 October 23; published 2010 December 3
}

\begin{abstract}
Isocyanic acid (HNCO) is a well-known interstellar molecule. Evidence also exists for the presence of two of its metastable isomers in the interstellar medium: HCNO (fulminic acid) and HOCN (cyanic acid). Fulminic acid has been detected toward cold and lukewarm sources, while cyanic acid has been detected both in these sources and in warm sources in the Galactic Center. Gas-phase models can reproduce the abundances of the isomers in cold sources, but overproduce HCNO in the Galactic Center. Here we present a detailed study of a gas-grain model that contains these three isomers, plus a fourth isomer, isofulminic acid (HONC), for four types of sources: hot cores, the warm envelopes of hot cores, lukewarm corinos, and cold cores. The current model is partially able to rationalize the abundances of $\mathrm{HNCO}, \mathrm{HOCN}$, and $\mathrm{HCNO}$ in cold and warm sources. Predictions for $\mathrm{HONC}$ in all environments are also made.
\end{abstract}

Key words: astrochemistry - ISM: abundances - ISM: molecules

\section{INTRODUCTION}

The well-known interstellar molecule isocyanic acid (HNCO) has been detected toward sources with a variety of physical conditions, including photon-dominated regions (Jansen et al. 1995), translucent clouds (Turner et al. 1999), cold dense cores (Brown 1981; Marcelino et al. 2009), hot cores (Churchwell et al. 1986; Snyder \& Buhl 1972; Martín et al. 2008), a "lukewarm corino" (Marcelino et al. 2009), and assorted clouds throughout the Galactic Center (Menten 2004; Martín et al. 2008; Turner 1991). The fractional abundance of HNCO with respect to molecular hydrogen varies somewhat in different sources. In the cold-core TMC-1, its fractional abundance is $5 \times 10^{-10}$ with respect to $\mathrm{H}_{2}$ (Marcelino et al. 2009), while in other cold sources it can be somewhat lower (Marcelino et al. 2010). In assorted sources in the giant cloud Sgr B2, the abundance of HNCO is $\geqslant 10^{-9}$ (Churchwell et al. 1986; Brünken et al. 2009a, 2010; Marcelino et al. 2010), although these abundances can be highly uncertain due to the uncertain $\mathrm{H}_{2}$ column density (Liu \& Snyder 1999). The metastable isomer of lowest energy, cyanic acid (HOCN), was identified in Sgr $\mathrm{B} 2(\mathrm{OH})$ with an estimated abundance relative to $\mathrm{HNCO}$ of 0.004 and an estimated fractional abundance of $1 \times 10^{-11}$ (Brünken et al. 2009a; Turner 1991). In addition, HOCN has also been detected in and around the hot cores Sgr B2(M) and Sgr B2(N), elsewhere in Sgr B2, in the hot corino IRAS 16293-2422, in the lukewarm corino L1527, and in a number of cold sources with an abundance of $\approx 0.003-0.03$ with respect to HNCO (Brünken et al. 2010; Marcelino et al. 2010). An even higher energy isomer, fulminic acid (HCNO), has been detected toward the cold sources in which HOCN was detected as well as toward the lukewarm corino L1527. The HCNO/ $\mathrm{HOCN}$ abundance ratio was estimated to be $\approx 1$ in these sources (Marcelino et al. 2009, 2010). On the other hand, a search for HCNO in warm regions such as the Sgr B2 sources, Orion, and even IRAS 16293-2422 ended in negative results, with upper limits to the $\mathrm{HCNO} / \mathrm{HOCN}$ abundance ratio ranging from 0.006 to 0.9 (Marcelino et al. 2009, 2010). Now that HONC has been studied in the laboratory (Mladenović et al. 2009), this highenergy isomer can also be searched for.

After HNCO was first detected toward Sgr B2 (Snyder \& Buhl 1972), a gas-phase synthesis was suggested by Iglesias (1977). The synthesis starts from the ion $\mathrm{NCO}^{+}$and consists of two ion-molecule reactions with $\mathrm{H}_{2}$ followed by dissociative recombination:

$$
\begin{gathered}
\mathrm{NCO}^{+}+\mathrm{H}_{2} \longrightarrow \mathrm{HNCO}^{+}+\mathrm{H}, \\
\mathrm{HNCO}^{+}+\mathrm{H}_{2} \longrightarrow \mathrm{HNCOH}^{+}+\mathrm{H} \\
\mathrm{HNCOH}^{+}+e^{-} \longrightarrow \mathrm{HNCO}+\mathrm{H}
\end{gathered}
$$

A similar synthesis was advocated by Brown (1981) to explain the abundance of HNCO in TMC-1, although he assumed the lower energy isomer $\mathrm{H}_{2} \mathrm{NCO}^{+}$to be the product in Reaction (2). Iglesias (1977) estimated the HNCO fractional abundance to be around $10^{-10}$ for a cold cloud at a density $n_{\mathrm{H}}=2 \times 10^{4} \mathrm{~cm}^{-3}$ with a lower fractional abundance at higher densities. Marcelino et al. (2009) reported a gas-phase model for HNCO, HOCN, and HCNO in a steady-state study of cold dense cores, adding considerably to the ions considered by Iglesias (1977). This network was extended and utilized at early time as well as steady state in Marcelino et al. (2010). In this network, HOCN can be formed from the precursor ion $\mathrm{HNCOH}^{+}$, whereas $\mathrm{HCNO}$ is formed primarily from the intensively studied neutral-neutral reaction between methylene and nitric oxide (Glarborg et al. 1998; Roggenbuck \& Temps 1998; Fikri et al. 2001; Eschenko et al. 2002):

$$
\mathrm{CH}_{2}+\mathrm{NO} \longrightarrow \mathrm{HCNO}+\mathrm{H} \text {. }
$$

The gas-phase theoretical results of Marcelino et al. (2010) are in reasonable agreement with the observed isomeric abundances in cold cores and in the lukewarm corino L1527. In the warm sources toward the Galactic Center, however, the predictions for the abundance of $\mathrm{HCNO}$ are far too large unless the products for Reaction (4) do not include $\mathrm{HCNO}$, a conclusion reached 
in quantum chemical studies by Zhang et al. (2004) but by few other authors. Our quantum chemical studies (see Section 2) support $\mathrm{HCNO}+\mathrm{H}$ as a product channel; the exothermicity of the reaction is $22.7 \mathrm{kcal} \mathrm{mol}^{-1}\left(1 \mathrm{kcal} \mathrm{mol}^{-1}=503 \mathrm{~K}\right)$, as originally calculated by Roggenbuck \& Temps (1998), with apparently no barrier.

In addition to gas-phase syntheses of $\mathrm{HNCO}, \mathrm{HOCN}$, and HCNO, syntheses on the surfaces of dust grains have been discussed recently by Brünken et al. (2010). It has been assumed for some time that $\mathrm{HNCO}$ can be produced efficiently on grain surfaces by the hydrogenation of accreted NCO (Hasegawa \& Herbst 1993; Garrod \& Herbst 2006). A recent detailed gasgrain model of the chemistry of HNCO in assorted environments concludes that surface routes are needed to account for its abundance (Tideswell et al. 2010). Another study concluded the same for HNCO production in the starburst galaxy NGC 253 (Martín et al. 2009). It is of course possible that HOCN can also be formed in this manner, while other surface routes could lead to the isomers HCNO and HONC. In cold sources, any of the isomers formed on dust particles would have to desorb into the gas in some non-thermal manner (e.g., photodesorption) to be detected, whereas in hot cores and their environments the ice mantles formed in prior cold eras evaporate at least partially.

In this work, we report results for all four isomers$\mathrm{HNCO}, \mathrm{HOCN}, \mathrm{HCNO}$, and $\mathrm{HONC}$ - using gas-grain simulations, which contain both gas-phase and grain-surface syntheses. For the different physical conditions of the various sources where these isomers were detected, we developed four models: a three-phase warm-up model for hot cores (Garrod \& Herbst 2006), a similar model for their slightly cooler surroundings, a lukewarm corino warm-up model for L1527 (Hassel et al. 2008), and a constant low-temperature $(10 \mathrm{~K})$ model for cold cores. Quantum chemical calculations have been performed to determine whether some unstudied but possibly important gasphase reactions are exothermic and barrierless. Some results of this study have already appeared in Marcelino et al. (2010).

The remainder of this work is organized as follows. In Section 2, we describe the quantum chemical methods used, the surface chemical processes leading to $\mathrm{HNCO}$ and its isomers (hereafter the CHNO isomers), the gas-grain network, and the physical parameters used in the four models, while in Section 3 we discuss our results and compare them with observations. The paper ends with our discussion in Section 4.

\section{QUANTUM CHEMICAL CALCULATIONS AND CHEMICAL REACTIONS}

Almost all new quantum chemical calculations reported here were undertaken by using the hybrid density functional B3LYP method (Becke 1993; Lee et al. 1988) with 6-311G(d,p) basis functions in order to obtain the molecular structures. Higherlevel calculations with the $\operatorname{CCSD}(\mathrm{T}) / \mathrm{aug}$-cc-pVTZ method were used to evaluate the relative energies of all species (Pople et al. 1987; Dunning 1989). Energies were corrected by zero-point vibrational energies calculated with the B3LYP/6$311 \mathrm{G}(\mathrm{d}, \mathrm{p})$ method without scaling. All calculations were undertaken with the GAUSSIAN 03 program package (Frisch et al. 2004).

In our study of the CHNO isomers, we used the Ohio State gas-grain network (Hasegawa et al. 1992; Hasegawa \& Herbst 1993; Ruffle \& Herbst 2001; Garrod \& Herbst 2006; Garrod et al. 2007; Hassel et al. 2008), which currently has almost 700 species, including 200 surface species, and over 6000 gasphase and grain-surface reactions. We have added more than
100 reactions concerning the formation and depletion of the CHNO isomers, most with estimated rates. The additional gasphase reactions are listed in Table 1, while the additional surface processes are listed in Table 2. In each table, the rate coefficients (Table 1) or parameters used in obtaining the rate coefficients (Table 2) are also listed. The surface reactions are considered to take place via the standard diffusive (Langmuir-Hinshelwood) mechanism on grains of radius $0.1 \mu \mathrm{m}$; the reactions are treated by rate equations (Herbst \& Millar 2008). Desorption energies $\left(E_{\mathrm{D}}\right)$ and diffusion energy barriers $\left(E_{\mathrm{b}}\right)$ for the surface species are set to the values discussed in Section 2.2 of Garrod \& Herbst (2006). For the CHNO isomers, we have estimated $E_{\mathrm{D}}$ to be $2800 \mathrm{~K}$ and $E_{\mathrm{b}}$ to be half of the desorption energy, based on similar species.

The gas-phase formation routes are similar to those discussed in Marcelino et al. (2009, 2010), so we focus on the formation routes on grain surfaces. We start from the isocyanate radical $\mathrm{NCO}$, which is formed in the gas mainly by the neutral-neutral reaction between atomic nitrogen and the formyl radical:

$$
\mathrm{N}+\mathrm{HCO} \longrightarrow \mathrm{NCO}+\mathrm{H} \text {. }
$$

The product radical can then be accreted onto the grains to form the species JNCO, where $\mathrm{J}$ designates a species in the icy grain mantle. Addition of a surface hydrogen atom leads to both isocyanic and cyanic acids:

$$
\begin{aligned}
& \mathrm{JH}+\mathrm{JNCO} \longrightarrow \mathrm{JHNCO}, \\
& \mathrm{JH}+\mathrm{JNCO} \longrightarrow \mathrm{JHOCN} .
\end{aligned}
$$

Subsequently, the acids can be desorbed into the gas, either by non-thermal mechanisms during a cold era or via sublimation during a warm-up or hot era. Non-thermal desorption mechanisms in the network include processes driven by cosmic rays, and the energy of exothermic reactions. The latter mechanism, in which the reaction exothermicity is statistically channeled into various exit channels via the use of unimolecular rate theory, is the dominant process (Garrod et al. 2007). To help destroy the grain species, surface photodissociation via external photons and cosmic-ray-produced photons is also included with rate coefficients equal to those that occur in the gas (Ruffle \& Herbst 2001). This assumption, though crude, is unavoidable given the lack of wavelength-dependent studies of photodissociation for surface species.

The surface synthesis of JHCNO starts from the radical CNO, a metastable isomer of $\mathrm{NCO}$, which is assumed to be produced on grain surfaces via the association of atomic carbon and nitrous oxide:

$$
\mathrm{JC}+\mathrm{JNO} \longrightarrow \mathrm{JCNO}
$$

after which hydrogenation produces the surface $\mathrm{HCNO}$ isomer:

$$
\mathrm{JH}+\mathrm{JCNO} \longrightarrow \mathrm{JHCNO} .
$$

Non-thermal desorption or sublimation then produces the gasphase species. The fourth isomer, HONC, is formed by a surface reaction analogous to Reaction (9); unlike HCNO, however, it is not formed by a gas-phase formation reaction analogous to Reaction (4) to the best of our knowledge.

Once produced, the gaseous CHNO isomers are depleted by accretion onto grains, by photodissociation involving mainly cosmic-ray-induced photons, and by chemical reactions with cations (e.g., $\mathrm{H}_{3}^{+}$) or possibly the abundant neutral atoms $\mathrm{C}$ and, 
Table 1

CHNO and Related Gas-phase Reactions

\begin{tabular}{|c|c|c|c|c|}
\hline Reaction & $\alpha^{\mathrm{a}}$ & $\beta$ & $\gamma$ & Reference \\
\hline \multicolumn{5}{|c|}{$\mathrm{HNCO}$} \\
\hline $\mathrm{HNCO} \rightarrow \mathrm{NH}+\mathrm{CO}(\mathrm{CRPh})$ & $6.00(+03)$ & 0 & 0 & 1 \\
\hline $\mathrm{H}^{+}+\mathrm{HNCO} \rightarrow \mathrm{NH}_{2}^{+}+\mathrm{CO}$ & $7.94(-09)$ & -0.5 & 0 & 1 \\
\hline $\mathrm{He}^{+}+\mathrm{HNCO} \rightarrow \mathrm{NCO}^{+}+\mathrm{H}+\mathrm{He}$ & $5.68(-09)$ & -0.5 & 0 & 1 \\
\hline $\mathrm{He}^{+}+\mathrm{HNCO} \rightarrow \mathrm{HNCO}^{+}+\mathrm{He}$ & $5.68(-09)$ & -0.5 & 0 & 2 \\
\hline $\mathrm{H}_{3}^{+}+\mathrm{HNCO} \rightarrow \mathrm{H}_{2} \mathrm{NCO}^{+}+\mathrm{H}_{2}$ & $3.69(-09)$ & -0.5 & 0 & 2 \\
\hline $\mathrm{H}_{3}^{+}+\mathrm{HNCO} \rightarrow \mathrm{HNCOH}^{+}+\mathrm{H}_{2}$ & $3.69(-09)$ & -0.5 & 0 & 2 \\
\hline $\mathrm{HNCO} \rightarrow \mathrm{NH}+\mathrm{CO}(h v)$ & $1.00(-09)$ & 0 & 1.7 & 2 \\
\hline $\mathrm{HNCO}+\mathrm{C} \rightarrow \mathrm{CO}+\mathrm{HNC}$ & $1.00(-12)$ & 0 & 0 & 2,8 \\
\hline $\mathrm{CH}_{2}+\mathrm{NO} \rightarrow \mathrm{HNCO}+\mathrm{H}$ & $3.65(-12)$ & 0 & 0 & $2,5,7$ \\
\hline \multicolumn{5}{|c|}{$\mathrm{HOCN}$} \\
\hline $\mathrm{HOCN} \rightarrow \mathrm{OH}+\mathrm{CN}(\mathrm{CRPh})$ & $6.00(+03)$ & 0 & 0 & 2 \\
\hline $\mathrm{H}^{+}+\mathrm{HOCN} \rightarrow \mathrm{H}_{2} \mathrm{O}^{+}+\mathrm{CN}$ & $6.25(-09)$ & -0.5 & 0 & 2 \\
\hline $\mathrm{He}^{+}+\mathrm{HOCN} \rightarrow \mathrm{NCO}^{+}+\mathrm{H}+\mathrm{He}$ & $4.47(-09)$ & -0.5 & 0 & 2 \\
\hline $\mathrm{H}_{3}^{+}+\mathrm{HOCN} \rightarrow \mathrm{HNCOH}^{+}+\mathrm{H}_{2}$ & $8.54(-09)$ & -0.5 & 0 & 2 \\
\hline $\mathrm{H}_{3}^{+}+\mathrm{HOCN} \rightarrow \mathrm{H}_{2} \mathrm{OCN}^{+}+\mathrm{H}_{2}$ & $8.54(-09)$ & -0.5 & 0 & 2 \\
\hline $\mathrm{HOCN} \rightarrow \mathrm{OH}+\mathrm{CN}(h v)$ & $1.00(-09)$ & 0 & 1.7 & 2 \\
\hline $\mathrm{HOCN}+\mathrm{O} \rightarrow \mathrm{OH}+\mathrm{NCO}$ & $3.33(-11)$ & 0 & 2470 & 2,8 \\
\hline $\mathrm{HOCN}+\mathrm{C} \rightarrow \mathrm{CO}+\mathrm{HCN}$ & $3.33(-11)$ & 0 & 0 & 2,8 \\
\hline
\end{tabular}

$\mathrm{HNCO}^{+}, \mathrm{HOCN}^{+}, \mathrm{H}_{2} \mathrm{NCO}^{+}, \mathrm{HNCOH}^{+}, \mathrm{H}_{2} \mathrm{OCN}^{+}$

\begin{tabular}{|c|c|c|c|c|}
\hline $\mathrm{NCO}+\mathrm{H}_{3}^{+} \rightarrow \mathrm{HNCO}^{+}+\mathrm{H}_{2}$ & $1.64(-08)$ & -0.5 & 0 & 1 \\
\hline $\mathrm{NCO}+\mathrm{H}_{3}^{+} \rightarrow \mathrm{HOCN}^{+}+\mathrm{H}_{2}$ & $1.64(-08)$ & -0.5 & 0 & 2 \\
\hline $\mathrm{NCO}^{+}+\breve{\mathrm{H}}_{2} \rightarrow \mathrm{HNCO}^{+}+\overrightarrow{\mathrm{H}}$ & $1.51(-09)$ & 0 & 0 & 3,4 \\
\hline $\mathrm{NCO}^{+}+\mathrm{H}_{2} \rightarrow \mathrm{HOCN}^{+}+\mathrm{H}$ & $1.51(-09)$ & 0 & 0 & 2 \\
\hline $\mathrm{HNCO}^{+}+\mathrm{H}_{2} \rightarrow \mathrm{H}_{2} \mathrm{NCO}^{+}+\mathrm{H}$ & $1.51(-09)$ & 0 & 0 & 3,4 \\
\hline $\mathrm{HNCO}^{+}+\mathrm{H}_{2} \rightarrow \mathrm{HNCOH}^{+}+\mathrm{H}$ & $1.51(-09)$ & 0 & 0 & 3,4 \\
\hline $\mathrm{HNCO}^{+}+e^{-} \rightarrow \mathrm{CO}+\mathrm{NH}$ & $1.50(-07)$ & -0.5 & 0 & 2 \\
\hline $\mathrm{HNCO}^{+}+e^{-} \rightarrow \mathrm{H}+\mathrm{NCO}$ & $1.50(-07)$ & -0.5 & 0 & 2 \\
\hline $\mathrm{H}_{2} \mathrm{NCO}^{+}+e^{-} \rightarrow \mathrm{HNCO}+\mathrm{H}$ & $1.50(-07)$ & -0.5 & 0 & 2 \\
\hline $\mathrm{H}_{2} \mathrm{NCO}^{+}+e^{-} \rightarrow \mathrm{NH}_{2}+\mathrm{CO}$ & $1.50(-07)$ & -0.5 & 0 & 2 \\
\hline $\mathrm{HOCN}^{+}+\mathrm{H}_{2} \rightarrow \mathrm{HNCOH}^{+}+\mathrm{H}$ & $1.51(-09)$ & 0 & 0 & 2 \\
\hline $\mathrm{HOCN}^{+}+\mathrm{H}_{2} \rightarrow \mathrm{H}_{2} \mathrm{OCN}^{+}+\mathrm{H}$ & $1.51(-09)$ & 0 & 0 & 2 \\
\hline $\mathrm{HOCN}^{+}+e^{-} \rightarrow \mathrm{OH}+\mathrm{CN}$ & $1.50(-07)$ & -0.5 & 0 & 2 \\
\hline $\mathrm{HOCN}^{+}+e^{-} \rightarrow \mathrm{H}+\mathrm{NCO}$ & $1.50(-07)$ & -0.5 & 0 & 2 \\
\hline $\mathrm{HNCOH}^{+}+e^{-} \rightarrow \mathrm{HNCO}+\mathrm{H}$ & $1.00(-07)$ & -0.5 & 0 & 2 \\
\hline $\mathrm{HNCOH}^{+}+e^{-} \rightarrow \mathrm{HOCN}+\mathrm{H}$ & $1.00(-07)$ & -0.5 & 0 & 2 \\
\hline $\mathrm{HNCOH}^{+}+e^{-} \rightarrow \mathrm{NH}+\mathrm{HCO}$ & $1.00(-07)$ & -0.5 & 0 & 2 \\
\hline $\mathrm{H}_{2} \mathrm{OCN}^{+}+e^{-} \rightarrow \mathrm{HOCN}+\mathrm{H}$ & $1.50(-07)$ & -0.5 & 0 & 2 \\
\hline $\mathrm{H}_{2} \mathrm{OCN}^{+}+e^{-} \rightarrow \mathrm{H}_{2} \mathrm{O}+\mathrm{CN}$ & $1.50(-07)$ & -0.5 & 0 & 2 \\
\hline \multicolumn{5}{|c|}{$\mathrm{HCNO}$} \\
\hline $\mathrm{HCNO} \rightarrow \mathrm{CH}+\mathrm{NO}(\mathrm{CRPh})$ & $6.00(+03)$ & 0 & 0 & 2 \\
\hline $\mathrm{H}^{+}+\mathrm{HCNO} \rightarrow \mathrm{CH}_{2}^{+}+\mathrm{NO}$ & $1.17(-08)$ & -0.5 & 0 & 2 \\
\hline $\mathrm{He}^{+}+\mathrm{HCNO} \rightarrow \mathrm{HCNO}^{+}+\mathrm{He}$ & $8.39(-09)$ & -0.5 & 0 & 2 \\
\hline $\mathrm{H}_{3}^{+}+\mathrm{HCNO} \rightarrow \mathrm{HCNOH}^{+}+\mathrm{H}_{2}$ & $6.92(-09)$ & -0.5 & 0 & 2 \\
\hline $\mathrm{H}_{3}^{+}+\mathrm{HCNO} \rightarrow \mathrm{H}_{2} \mathrm{CNO}^{+}+\mathrm{H}_{2}$ & $6.92(-09)$ & -0.5 & 0 & 2 \\
\hline $\mathrm{HCNO}+\mathrm{C} \rightarrow \mathrm{C}_{2} \mathrm{H}+\mathrm{NO}$ & $3.33(-11)$ & 0 & 0 & 2,8 \\
\hline $\mathrm{HCNO}+\mathrm{O} \rightarrow \mathrm{CO}+\mathrm{HNO}$ & $3.33(-11)$ & 0 & 195 & 2,6 \\
\hline $\mathrm{CH}_{2}+\mathrm{NO} \rightarrow \mathrm{HCNO}+\mathrm{H}$ & $3.65(-11)$ & 0 & 0 & $2,5,7$ \\
\hline $\mathrm{HCNO} \rightarrow \mathrm{CH}+\mathrm{NO}(h v)$ & $1.00(-9)$ & 0 & 1.7 & 2 \\
\hline \multicolumn{5}{|c|}{ HONC } \\
\hline $\mathrm{HONC} \rightarrow \mathrm{CN}+\mathrm{OH}(\mathrm{CRPh})$ & $6.00(+03)$ & 0 & 0 & 2 \\
\hline $\mathrm{H}^{+}+\mathrm{HONC} \rightarrow \mathrm{H}_{2} \mathrm{O}^{+}+\mathrm{CN}$ & $1.45(-08)$ & -0.5 & 0 & 2 \\
\hline $\mathrm{He}^{+}+\mathrm{HONC} \rightarrow \mathrm{HONC}^{+}+\mathrm{He}$ & $1.03(-08)$ & -0.5 & 0 & 2 \\
\hline $\mathrm{H}_{3}^{+}+\mathrm{HONC} \rightarrow \mathrm{HCNOH}^{+}+\mathrm{H}_{2}$ & $8.54(-09)$ & -0.5 & 0 & 2 \\
\hline $\mathrm{HONC}+\mathrm{O} \rightarrow \mathrm{OH}+\mathrm{CNO}$ & $3.33(-11)$ & 0 & 3570 & 2,8 \\
\hline $\mathrm{HONC}+\mathrm{C} \rightarrow \mathrm{OH}+\mathrm{C}_{2} \mathrm{~N}$ & $3.33(-11)$ & 0 & 0 & 2,8 \\
\hline $\mathrm{HONC} \rightarrow \mathrm{OH}+\mathrm{CN}(h v)$ & $1.00(-09)$ & 0 & 1.7 & 2 \\
\hline
\end{tabular}

\begin{tabular}{llcccc}
\hline \multicolumn{5}{c}{$\mathrm{HCNO}^{+}, \mathrm{HONC}^{+}, \mathrm{HCNOH}^{+}, \mathrm{H}_{2} \mathrm{CNO}^{+}$} \\
\hline $\mathrm{HCNO}^{+}+\mathrm{H}_{2} \rightarrow \mathrm{HCNOH}^{+}+\mathrm{H}$ & $1.51(-09)$ & 0 & 0 & 2 \\
$\mathrm{HCNO}^{+}+e^{-} \rightarrow \mathrm{CH}+\mathrm{NO}$ & $1.50(-07)$ & -0.5 & 0 & 2 \\
$\mathrm{HCNO}^{+}+e^{-} \rightarrow \mathrm{H}+\mathrm{CNO}$ & $1.50(-07)$ & -0.5 & 0 & 2
\end{tabular}

Table 1

(Continued)

\begin{tabular}{|c|c|c|c|c|}
\hline Reaction & $\alpha^{\mathrm{a}}$ & $\beta$ & $\gamma$ & Reference \\
\hline $\mathrm{HCNOH}^{+}+e^{-} \rightarrow \mathrm{HCNO}+\mathrm{H}$ & $1.00(-07)$ & -0.5 & 0 & 2 \\
\hline $\mathrm{HCNOH}^{+}+e^{-} \rightarrow \mathrm{HCN}+\mathrm{OH}$ & $1.00(-07)$ & -0.5 & 0 & 2 \\
\hline $\mathrm{H}_{2} \mathrm{CNO}^{+}+e^{-} \rightarrow \mathrm{HCNO}+\mathrm{H}$ & $1.50(-07)$ & -0.5 & 0 & 2 \\
\hline $\mathrm{H}_{2} \mathrm{CNO}^{+}+e^{-} \rightarrow \mathrm{C}_{2} \mathrm{H}+\mathrm{NO}$ & $1.50(-07)$ & -0.5 & 0 & 2 \\
\hline $\mathrm{HCNO}^{+}+\mathrm{H}_{2} \rightarrow \mathrm{HCNOH}^{+}+\mathrm{H}$ & $1.51(-09)$ & 0 & 0 & 2 \\
\hline $\mathrm{HCNO}^{+}+\mathrm{H}_{2} \rightarrow \mathrm{H}_{2} \mathrm{CNO}^{+}+\mathrm{H}$ & $1.51(-09)$ & 0 & 0 & 2 \\
\hline $\mathrm{HONC}^{+}+\mathrm{H}_{2} \rightarrow \mathrm{HCNOH}^{+}+\mathrm{H}$ & $1.51(-09)$ & 0 & 0 & 2 \\
\hline $\mathrm{HONC}^{+}+e^{-} \rightarrow \mathrm{OH}+\mathrm{CN}$ & $1.50(-07)$ & -0.5 & 0 & 2 \\
\hline $\mathrm{HONC}^{+}+e^{-} \rightarrow \mathrm{H}+\mathrm{CNO}$ & $1.50(-07)$ & -0.5 & 0 & 2 \\
\hline $\mathrm{HCNOH}^{+}+e^{-} \rightarrow \mathrm{HONC}+\mathrm{H}$ & $1.00(-07)$ & -0.5 & 0 & 2 \\
\hline \multicolumn{5}{|c|}{$\mathrm{NCO}, \mathrm{CNO}$} \\
\hline $\mathrm{H}+\mathrm{NCO} \rightarrow \mathrm{OH}+\mathrm{CN}$ & $1.00(-10)$ & 0 & 0 & 2 \\
\hline $\mathrm{H}+\mathrm{CNO} \rightarrow \mathrm{OH}+\mathrm{CN}$ & $1.00(-10)$ & 0 & 0 & 2 \\
\hline $\mathrm{NCO} \rightarrow \mathrm{CN}+\mathrm{O}(\mathrm{CRPh})$ & $1.5(+03)$ & 0 & 0 & 1 \\
\hline $\mathrm{CNO} \rightarrow \mathrm{CN}+\mathrm{O}(\mathrm{CRPh})$ & $1.5(+03)$ & 0 & 0 & 2 \\
\hline $\mathrm{C}^{+}+\mathrm{NCO} \rightarrow \mathrm{CO}^{+}+\mathrm{CN}$ & $1.90(-09)$ & -0.5 & 0 & 1 \\
\hline $\mathrm{He}^{+}+\mathrm{NCO} \rightarrow \mathrm{O}^{+}+\mathrm{CN}+\mathrm{He}$ & $1.50(-09)$ & -0.5 & 0 & 1 \\
\hline $\mathrm{He}^{+}+\mathrm{NCO} \rightarrow \mathrm{CN}^{+}+\mathrm{O}+\mathrm{He}$ & $1.50(-09)$ & -0.5 & 0 & 1 \\
\hline $\mathrm{C}^{+}+\mathrm{CNO} \rightarrow \mathrm{CO}^{+}+\mathrm{CN}$ & $8.98(-09)$ & -0.5 & 0 & 2 \\
\hline $\mathrm{He}^{+}+\mathrm{CNO} \rightarrow \mathrm{O}^{+}+\mathrm{CN}+\mathrm{He}$ & $1.99(-08)$ & -0.5 & 0 & 2 \\
\hline $\mathrm{He}^{+}+\mathrm{CNO} \rightarrow \mathrm{CN}^{+}+\mathrm{O}+\mathrm{He}$ & $1.99(-08)$ & -0.5 & 0 & 2 \\
\hline $\mathrm{H}_{3}^{+}+\mathrm{CNO} \rightarrow \mathrm{HCNO}^{+}+\mathrm{H}_{2}$ & $1.64(-08)$ & -0.5 & 0 & 2 \\
\hline $\mathrm{H}_{3}^{+}+\mathrm{CNO} \rightarrow \mathrm{HONC}^{+}+\mathrm{H}_{2}$ & $1.64(-08)$ & -0.5 & 0 & 2 \\
\hline $\mathrm{C}+\mathrm{NCO} \rightarrow \mathrm{CO}+\mathrm{CN}$ & $1.00(-10)$ & 0 & 0 & 1 \\
\hline $\mathrm{O}+\mathrm{NCO} \rightarrow \mathrm{CO}+\mathrm{NO}$ & $1.00(-10)$ & 0 & 0 & 1 \\
\hline $\mathrm{O}+\mathrm{CNO} \rightarrow \mathrm{CO}+\mathrm{NO}$ & $1.00(-10)$ & 0 & 0 & 2 \\
\hline $\mathrm{N}+\mathrm{HCO} \rightarrow \mathrm{NCO}+\mathrm{H}$ & $1.00(-10)$ & 0 & 0 & 1 \\
\hline $\mathrm{CH}+\mathrm{NO} \rightarrow \mathrm{CNO}+\mathrm{H}$ & $1.00(-10)$ & 0 & 0 & 1,2 \\
\hline $\mathrm{NCO} \rightarrow \mathrm{CN}+\mathrm{O}(h v)$ & $1.00(-11)$ & 0 & 2.0 & 1 \\
\hline $\mathrm{CNO} \rightarrow \mathrm{CN}+\mathrm{O}(h v)$ & $1.00(-11)$ & 0 & 2.0 & 2 \\
\hline
\end{tabular}

Notes. $a(-b)$ signifies $a \times 10^{-b}$.

a Bimolecular rate coefficients are tabulated as $\alpha \times(T / 300)^{\beta} \times e^{-\gamma / T}$ in units of $\mathrm{cm}^{3} \mathrm{~s}^{-1}$. Photodissociation rate coefficients are tabulated as $\alpha \times$ $(T / 300)^{\beta} \times e^{-\gamma A_{v}}$ in units of $\mathrm{s}^{-1}$. Rate coefficients for cosmic-ray-induced photodissociation are tabulated in terms of $\zeta\left(\mathrm{s}^{-1}\right)$.

References. (1) OSU gas-grain network (Hasegawa et al. 1992; Garrod et al. 2007; Hassel et al. 2008); (2) estimation according to analogous reaction rates; (3) Iglesias 1977; (4) Brown 1981; (5) Glarborg et al. 1998; (6) Feng \& Hershberger 2007; (7) Fikri et al. 2001; (8) this work, based on potential and barrier calculations by Osamura.

most importantly, O. Since reaction by neutral atoms can be the dominant destructive mechanism for the CHNO isomers, if it occurs exothermically and without an activation energy barrier, we used quantum chemical techniques to investigate some of these reactions. Unfortunately, the large number of reaction channels means that we have not been able to perform all of the necessary calculations, so we have had to make some estimates as well.

For HNCO, the lowest energy isomer (Schuurman et al. 2004), all the reaction channels with $O$ have an energy barrier and can be neglected despite the fact that two of the channels$\mathrm{HNO}+\mathrm{CO}$ and $\mathrm{NH}+\mathrm{CO}_{2}-$ are calculated to be exothermic. We calculate that the reaction between $\mathrm{HNCO}$ and $\mathrm{C}$ has five sets of exothermic products. In particular, we have shown that the product channel $\mathrm{CO}+\mathrm{HNC}$ with $\mathrm{HNC}$ in its excited triplet state is exothermic by $15.7 \mathrm{kcal} \mathrm{mol}^{-1}\left(1 \mathrm{kcal} \mathrm{mol}^{-1}=503 \mathrm{~K}\right)$ and free of any activation energy barrier. We assume a temperatureindependent rate coefficient of $1 \times 10^{-12} \mathrm{~cm}^{3} \mathrm{~s}^{-1}$ for this channel, which is the only one included in our network at present since we do not know if the other exothermic channels 
Table 2

CHNO and Related Surface Reactions

\begin{tabular}{|c|c|c|c|}
\hline \multirow{2}{*}{$\begin{array}{l}\text { Reaction } \\
\text { Surface reactions }\end{array}$} & \multicolumn{2}{|c|}{ Parameters $^{\mathrm{a}}$} & \multirow[t]{2}{*}{ Reference } \\
\hline & $E_{\mathrm{b}, \mathrm{A}}(\mathrm{K})$ & $E_{\mathrm{b}, \mathrm{B}}(\mathrm{K})$ & \\
\hline $\mathrm{JH}+\mathrm{JNCO} \rightarrow \mathrm{JHNCO}$ & 225 & 1200 & 1 \\
\hline $\mathrm{JH}+\mathrm{JNCO} \rightarrow \mathrm{JHOCN}$ & 225 & 1200 & 1,2 \\
\hline $\mathrm{JC}+\mathrm{JHOCN} \rightarrow \mathrm{JCO}+\mathrm{JHCN}$ & 400 & 1400 & 1,2 \\
\hline $\mathrm{JH}+\mathrm{JCNO} \rightarrow \mathrm{JHCNO}$ & 225 & 1200 & 1,2 \\
\hline $\mathrm{JC}+\mathrm{JHCNO} \rightarrow \mathrm{JC}_{2} \mathrm{H}+\mathrm{JNO}$ & 400 & 1400 & 1,2 \\
\hline $\mathrm{JH}+\mathrm{JCNO} \rightarrow \mathrm{JHONC}$ & 225 & 1200 & 1,2 \\
\hline $\mathrm{JO}+\mathrm{JHONC} \rightarrow \mathrm{JO}_{2} \mathrm{H}+\mathrm{JCN}$ & 400 & 1400 & 1,2 \\
\hline $\mathrm{JC}+\mathrm{JNCO} \rightarrow \mathrm{JCN}+\mathrm{JCO}$ & 400 & 1200 & 1 \\
\hline $\mathrm{JO}+\mathrm{JNCO} \rightarrow \mathrm{JCO}+\mathrm{JNO}$ & 400 & 1200 & 1,2 \\
\hline $\mathrm{JC}+\mathrm{JCNO} \rightarrow \mathrm{JCN}+\mathrm{JCO}$ & 400 & 1200 & 1,2 \\
\hline $\mathrm{JC}+\mathrm{JNO} \rightarrow \mathrm{JCNO}$ & 400 & 800 & 1,2 \\
\hline Reaction-induced desorption & $E_{\mathrm{D}}(\mathrm{K})$ & $E_{\text {exo }}(\mathrm{K})$ & \\
\hline $\mathrm{JH}+\mathrm{JNCO} \rightarrow \mathrm{HNCO}$ & 2800 & 53800 & 1 \\
\hline $\mathrm{JH}+\mathrm{JNCO} \rightarrow \mathrm{HOCN}$ & 2800 & 43100 & 2 \\
\hline $\mathrm{JC}+\mathrm{JHOCN} \rightarrow \mathrm{CO}+\mathrm{HCN}$ & 2050 & 81700 & 2 \\
\hline $\mathrm{JH}+\mathrm{JCNO} \rightarrow \mathrm{HCNO}$ & 2800 & 54000 & 2 \\
\hline $\mathrm{JC}+\mathrm{JHCNO} \rightarrow \mathrm{C}_{2} \mathrm{H}+\mathrm{NO}$ & 2140 & 28000 & 2 \\
\hline $\mathrm{JH}+\mathrm{JONC} \rightarrow \mathrm{HONC}$ & 2800 & 9440 & 2 \\
\hline $\mathrm{JO}+\mathrm{JHONC} \rightarrow \mathrm{O}_{2} \mathrm{H}+\mathrm{CN}$ & 3650 & 5710 & 2 \\
\hline $\mathrm{JC}+\mathrm{JNCO} \rightarrow \mathrm{CN}+\mathrm{CO}$ & 1600 & 62500 & 1 \\
\hline $\mathrm{JO}+\mathrm{JNCO} \rightarrow \mathrm{CO}+\mathrm{NO}$ & 1600 & 47800 & 2 \\
\hline $\mathrm{JCNO}+\mathrm{JC} \rightarrow \mathrm{CN}+\mathrm{CO}$ & 1600 & 95500 & 2 \\
\hline $\mathrm{JC}+\mathrm{JNO} \rightarrow \mathrm{CNO}$ & 2400 & 48800 & 2 \\
\hline Thermal and cosmic-ray desorption & $E_{\mathrm{D}}(\mathrm{K})$ & & \\
\hline $\mathrm{JHNCO} \rightarrow \mathrm{HNCO}$ & 2800 & & 1 \\
\hline $\mathrm{JHOCN} \rightarrow \mathrm{HOCN}$ & 2800 & & 2 \\
\hline $\mathrm{JHCNO} \rightarrow \mathrm{HCNO}$ & 2800 & & 2 \\
\hline $\mathrm{JHONC} \rightarrow \mathrm{HONC}$ & 2800 & & 2 \\
\hline $\mathrm{JCNO} \rightarrow \mathrm{CNO}$ & 2400 & & 2 \\
\hline Cosmic-ray-induced photodissociation & $\alpha\left(\mathrm{s}^{-1}\right)$ & & \\
\hline $\mathrm{JHNCO} \rightarrow \mathrm{JNH}+\mathrm{JCO}$ & $6.00(+03) \zeta$ & & 1 \\
\hline $\mathrm{JHOCN} \rightarrow \mathrm{JOH}+\mathrm{JCN}$ & $6.00(+03) \zeta$ & & 2 \\
\hline $\mathrm{JHCNO} \rightarrow \mathrm{JCH}+\mathrm{JNO}$ & $3.00(+03) \zeta$ & & 2 \\
\hline $\mathrm{JHONC} \rightarrow \mathrm{JOH}+\mathrm{JCN}$ & $3.00(+03) \zeta$ & & 2 \\
\hline $\mathrm{JCNO} \rightarrow \mathrm{JCN}+\mathrm{JO}$ & $3.00(+03) \zeta$ & & 2 \\
\hline Photodissociation & $\alpha\left(\mathrm{s}^{-1}\right)$ & $\gamma$ & \\
\hline $\mathrm{JHNCO} \rightarrow \mathrm{JNH}+\mathrm{JCO}$ & $1.00(-09)$ & 1.7 & 1 \\
\hline $\mathrm{JHOCN} \rightarrow \mathrm{JOH}+\mathrm{JCN}$ & $1.00(-09)$ & 1.7 & 2 \\
\hline $\mathrm{JHCNO} \rightarrow \mathrm{JCH}+\mathrm{JNO}$ & $1.00(-09)$ & 1.7 & 2 \\
\hline $\mathrm{JHONC} \rightarrow \mathrm{JOH}+\mathrm{JCN}$ & $1.00(-09)$ & 1.7 & 2 \\
\hline $\mathrm{JCNO} \rightarrow \mathrm{JCN}+\mathrm{JO}$ & $1.00(-11)$ & 2.0 & 2 \\
\hline
\end{tabular}

Notes. $a(-b)$ signifies $a \times 10^{-b}$.

a The parameters and how they are used in the formulae for the rate coefficients for assorted processes can be found in Hasegawa et al. (1992), Hasegawa \& Herbst (1993), Ruffle \& Herbst (2001), Garrod et al. (2007), and Herbst \& Millar (2008).

References. (1) OSU gas-grain network (Hasegawa et al. 1992; Garrod et al. 2007; Hassel et al. 2008); (2) estimation.

possess potential barriers. Given the number of possible product channels, both exothermic and endothermic, it is likely that the channel included possesses a rate coefficient somewhat lower than the gas kinetic value. The destruction path for HNCO with atomic carbon was not considered in previous models, and the lowered abundance of HNCO due to its inclusion is minimized by this choice of rate coefficient.

For the case of HOCN, which lies higher in energy than HNCO by $25 \mathrm{kcal} \mathrm{mol}^{-1}$ according to Schuurman et al. (2004) and by $24.2 \mathrm{kcal} \mathrm{mol}^{-1}$ from our calculations, the reaction with atomic carbon was found to be exothermic for eight sets of products, including $\mathrm{HCN}+\mathrm{CO}$ with $\mathrm{HCN}$ in its ground singlet state and its excited triplet state, but barrier calculations were not undertaken for most of these, nor was intersystem crossing studied for necessary spin flips. We have calculated a barrier of $15.9 \mathrm{kcal} \mathrm{mol}^{-1}$ for the product channel $\mathrm{CH}+\mathrm{NCO}$. The product channels $\mathrm{OH}+\mathrm{CNC}$ and $\mathrm{CNCO}+\mathrm{H}$ have been found to have no energy barriers, but the $\mathrm{OH}+\mathrm{CNC}$ channel is calculated to be $3.1 \mathrm{kcal} \mathrm{mol}^{-1}$ endothermic, while the $\mathrm{CNCO}+$ $\mathrm{H}$ channel is exothermic by $44.5 \mathrm{kcal} \mathrm{mol}^{-1}$. Since $\mathrm{CNCO}$ is not in our network, we included the exothermic $\mathrm{HCN}+\mathrm{CO}$ product channel so as to have a product channel without a barrier.

The reaction with atomic oxygen to produce the products $\mathrm{OH}$ and $\mathrm{NCO}$,

$$
\mathrm{HOCN}+\mathrm{O} \longrightarrow \mathrm{OH}+\mathrm{NCO},
$$

has been found to be exothermic with a large barrier of $4.9 \mathrm{kcal} \mathrm{mol}^{-1}(2460 \mathrm{~K})$ so that it is essentially unreactive. Other exothermic channels are assumed to have high barriers as well.

For the case of $\mathrm{HCNO}$, which lies $70 \mathrm{kcal} \mathrm{mol}^{-1}$ higher in energy than HNCO according to Schuurman et al. (2004) and $68.2 \mathrm{kcal} \mathrm{mol}^{-1}$ according to our calculations, the exothermic reaction with atomic oxygen has been previously studied. Laboratory results show that it has a barrier of $195 \pm 120 \mathrm{~K}$ and that products including CO dominate (Feng \& Hershberger 2007). On the other hand, theoretical results (Miller et al. 1998, 2003) indicate that no barrier exists for rapid reactions to form $\mathrm{HCO}+\mathrm{NO}$ and $\mathrm{NCO}+\mathrm{OH}$. We have chosen to use the laboratory results. Of the reactions with atomic carbon, the process

$$
\mathrm{C}+\mathrm{HCNO} \longrightarrow \mathrm{C}_{2} \mathrm{H}+\mathrm{NO}
$$

has been found to be exothermic by $47.8 \mathrm{kcal} \mathrm{mol}^{-1}$ and to have no energy barrier. We have included it in our reaction network.

For the highest energy isomer, HONC, with an energy relative to HNCO of $85 \mathrm{kcal} \mathrm{mol}^{-1}$ according to Schuurman et al. (2004) and $82.3 \mathrm{kcal} \mathrm{mol}^{-1}$ according to our calculations, we calculate that there is no attractive potential surface for reactions with atomic oxygen, which means that there must be energy barriers in order to produce any products. The lowest energy pathway appears to be associated with the reaction

$$
\mathrm{O}+\mathrm{HONC} \longrightarrow \mathrm{OH}+\mathrm{NCO}
$$

which has a barrier of $7.1 \mathrm{kcal} \mathrm{mol}^{-1}$, a value high enough that we can exclude the reaction from our model. We have also calculated that $\mathrm{HONC}$ reacts exothermically and without barrier with atomic carbon to form two sets of products: $\mathrm{OH}+\mathrm{CCN}$ (53.2 kcal mol $\mathrm{k}^{-1}$ exothermic) and $\mathrm{H}+\mathrm{CCNO}\left(19.6 \mathrm{kcal} \mathrm{mol}^{-1}\right.$ exothermic). The first product channel is included in our network, while the second is not since CCNO is not in the network.

Exothermic destruction reactions involving atomic hydrogen, namely

$$
\mathrm{H}+\mathrm{HOCN} \longrightarrow \mathrm{HNCO}+\mathrm{H}
$$

and

$$
\mathrm{H}+\mathrm{HONC} \longrightarrow \mathrm{HCNO}+\mathrm{H},
$$

were included in our initial treatment (Marcelino et al. 2010), but have been removed for this work since the analogous reaction

$$
\mathrm{HNC}+\mathrm{H} \longrightarrow \mathrm{HCN}+\mathrm{H}
$$




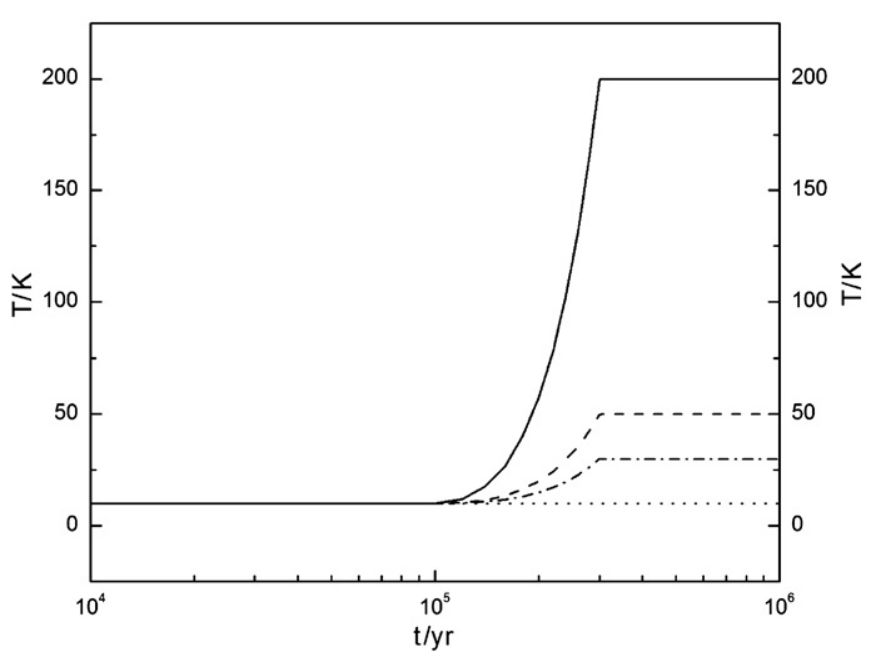

Figure 1. Thermal history of four gas-grain models: solid line-hot-core model; dashed line—warm-envelope model; dash-dotted line—lukewarm-core model; dotted line-cold-core model.

Table 3

Physical Parameters of the Models

\begin{tabular}{lcccc}
\hline \hline Parameter & Hot Core & Warm Envelope & Lukewarm & Cold Core \\
\hline$n_{\mathrm{H}}\left(\mathrm{cm}^{-3}\right)$ & $2 \times 10^{6}$ & $2 \times 10^{5}$ & $2 \times 10^{6}$ & $2 \times 10^{4}$ \\
$T_{\text {asymp }}(\mathrm{K})$ & 200 & 50 & 30 & 10 \\
$\zeta\left(\mathrm{s}^{-1}\right)$ & $1.3 \times 10^{-17}$ & $1.3 \times 10^{-17}$ & $1.3 \times 10^{-17}$ & $1.3 \times 10^{-17}$ \\
$A_{\mathrm{V}}$ & 10 & 10 & 10 & 10 \\
$d / g$ & 0.01 & 0.01 & 0.01 & 0.01 \\
$a_{\text {RRK }}$ & 0.01 & 0.01 & 0.01 & 0.01 \\
\hline
\end{tabular}

has been calculated to have a barrier of $\approx 4 \mathrm{kcal} \mathrm{mol}^{-1}(2000 \mathrm{~K})$ (Talbi et al. 1996). New calculations at the B3LYP/6-311G(d,p) level confirm that a barrier of $4.1 \mathrm{kcal} \mathrm{mol}^{-1}$ does indeed exist for $\mathrm{H}+\mathrm{HOCN}$. The barrier is expected to be even higher at the $\operatorname{CCSD}(\mathrm{T})$ level.

\subsection{Gas-grain Models}

To simulate the very different circumstances of hot, lukewarm, and cold cores, we have run four gas-grain models, two warm-up models based on our previous work on hot cores and corinos (Garrod \& Herbst 2006; Hassel et al. 2008), one warm-up model based on the lukewarm corino model for L1527 (Hassel et al. 2008), and one cold-core ( $T=10 \mathrm{~K}$ ) model based on the work of Garrod et al. (2007). The warm-up models consist of three phases: an initial cold phase at $10 \mathrm{~K}$ lasting for $10^{5} \mathrm{yr}$, during which both gas-phase and grain-surface chemistry occur, a warm-up phase lasting for $2 \times 10^{5} \mathrm{yr}$ during which the surface chemistry can produce large organic molecules before the ice mantle fully sublimes, and a hot-core phase, during which gas-phase chemistry acts to deplete the large organic molecules produced in the warm-up and cold phases. The length of the cold phase is sufficiently long for the higher density models so that most heavy material has condensed out onto the dust particles. The temperature increase during the actual warm-up phase occurs quadratically with time (Garrod \& Herbst 2006). The length of this phase is that estimated for a star of medium mass (Garrod \& Herbst 2006). The details of the warm up and its relation to the mass of the star being formed are discussed in Viti et al. (2004) and Garrod \& Herbst (2006).

The constant densities, final temperatures, and other model parameters of all four models are listed in Table 3 ; the parameters

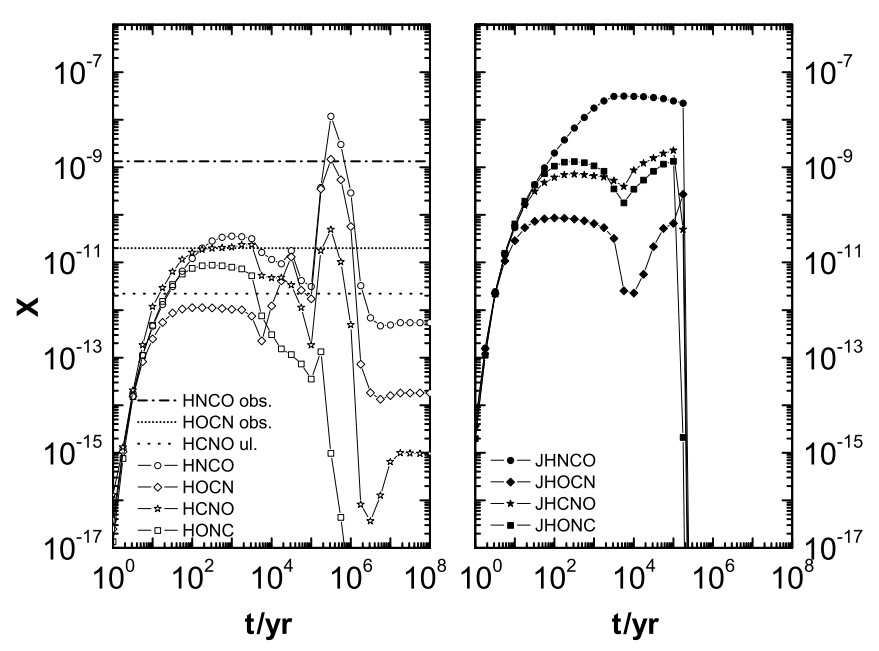

Figure 2. Calculated fractional abundances with respect to $n_{\mathrm{H}_{2}}$ for CHNO isomers in the gas (left panel, open symbols) and on dust particles (right panel, solid symbols) as functions of time in the hot-core model. Horizontal lines in the left panel indicate observed abundances for $\mathrm{HNCO}$ and $\mathrm{HOCN}$ and the upper limit for HCNO for the hot-core Sgr B2(M) (Marcelino et al. 2010).

Table 4

Initial Fractional Abundances

\begin{tabular}{lc}
\hline \hline Species & Abundance \\
\hline $\mathrm{He}$ & $6.00 \times 10^{-2}$ \\
$\mathrm{~N}$ & $2.14 \times 10^{-5}$ \\
$\mathrm{O}$ & $1.76 \times 10^{-4}$ \\
$\mathrm{H}_{2}$ & $5.00 \times 10^{-1}$ \\
$\mathrm{C}^{+}$ & $7.30 \times 10^{-5}$ \\
$\mathrm{~S}^{+}$ & $8.00 \times 10^{-8}$ \\
$\mathrm{Si}^{+}$ & $8.00 \times 10^{-9}$ \\
$\mathrm{Fe}^{+}$ & $3.00 \times 10^{-9}$ \\
$\mathrm{Na}^{+}$ & $2.00 \times 10^{-9}$ \\
$\mathrm{Mg}^{+}$ & $7.00 \times 10^{-9}$ \\
$\mathrm{P}^{+}$ & $3.00 \times 10^{-9}$ \\
$\mathrm{Cl}^{+}$ & $4.00 \times 10^{-9}$ \\
\hline
\end{tabular}

$\zeta, d / g$, and $a_{\mathrm{RRK}}$ refer, respectively, to the cosmic-ray ionization rate for $\mathrm{H}_{2}$, the dust-to-gas mass ratio, and a parameter that regulates the rate of desorption caused by exothermic reactions (Garrod et al. 2007). A plot of temperature versus time for the models is shown in Figure 1. The chosen initial abundances are listed in Table 4; these are based on the oxygen-rich low-metal abundances commonly used in our studies.

\section{RESULTS}

Calculated results for the CHNO isomers for the hot-core model, the warm-envelope model, the lukewarm model, and the cold-core model are shown in Figures 2, 3, 4, 5, and 6. In general, the lowest energy isomer, HNCO, is calculated to have the largest abundance by a significant factor, in agreement with observation, and the abundances for the detected metastable isomers $\mathrm{HOCN}$ and $\mathrm{HCNO}$ are often in reasonable agreement with observation for a significant period of time.

In Figure 2, we compare calculated hot-core results with observational abundances for $\mathrm{HNCO}$ and $\mathrm{HOCN}$ of $\approx 2 \times 10^{-9}$ and $\approx 2 \times 10^{-11}$, respectively, toward Sgr B2(M) (Marcelino et al. 2010). Much higher abundances of $\approx 2 \times 10^{-7}$ and $\approx 5 \times 10^{-10}$ are estimated toward Sgr B2(N) (Marcelino et al. 2010). We see that during the cold stage of the hot-core model, when the temperature remains at $10 \mathrm{~K}$, the calculated abundance 


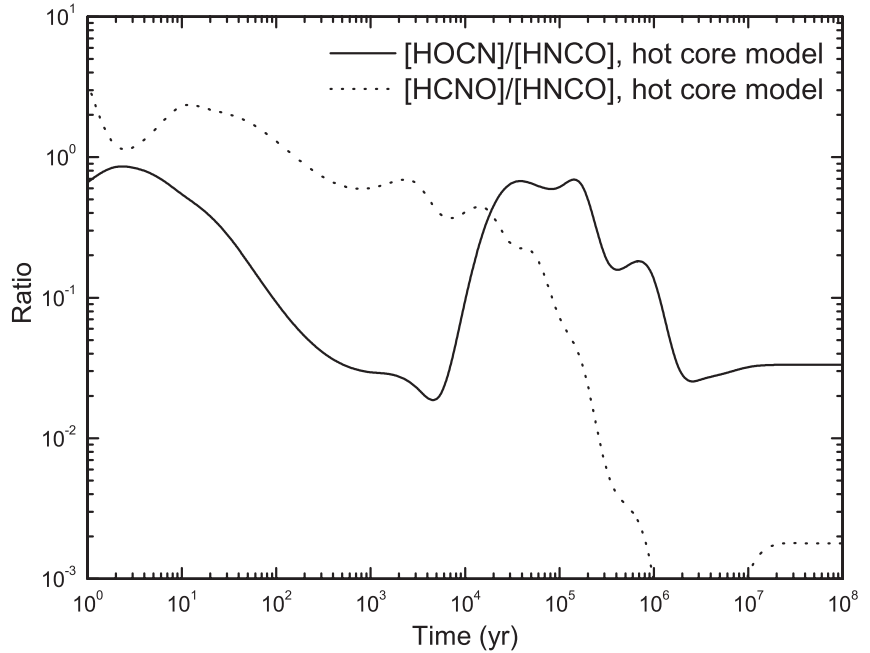

Figure 3. Calculated abundance ratios $\mathrm{HOCN} / \mathrm{HNCO}$ and $\mathrm{HCNO} / \mathrm{HNCO}$ in the gas as functions of time in the hot-core model.
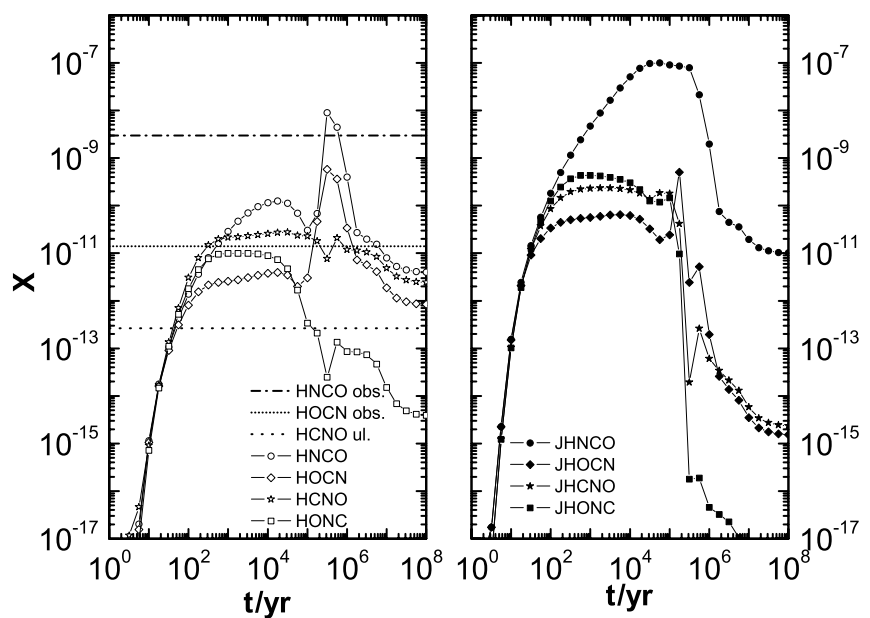

Figure 4. Same as Figure 2 except that the warm-envelope model is used. The observed $\mathrm{HNCO}$ and $\mathrm{HOCN}$ fractional abundances are toward Sgr B2(OH) (Churchwell et al. 1986; Brünken et al. 2009a). Similar fractional abundances are observed toward the warm environments of Sgr B2(M) and (N) (Marcelino et al. 2010; Brünken et al. 2010). The upper limit for HCNO has been obtained for the warm environment of the former.

of HNCO lies well below its observed value, while that of $\mathrm{HOCN}$ is in reasonable (order of magnitude) agreement with observation for a short period after $10^{4} \mathrm{yr}$. After the warmup stage begins at $10^{5} \mathrm{yr}$, a sharp decrease in the solid phase abundances due to sublimation (see the right panel) results in a sharp increase in the gas-phase abundances of three isomers, HNCO, HOCN, and HCNO, which peak near the end of the warm-up period, where the temperature is $200 \mathrm{~K}$. At this brief period, the calculated abundance of the dominant isomer $\mathrm{HNCO}$ lies about a factor of 10 greater than the observed abundance in Sgr B2(M) and a similar factor below the observed abundance in Sgr B2(N), while the calculated abundance of HOCN lies a factor of 50 above observation in Sgr B2(M) and a factor of 2 above observation in Sgr B2(N). In the final stage of the chemistry, during which the temperature remains at $200 \mathrm{~K}$, the gas-phase abundances of the isomers decrease because of the gas-phase chemistry. The best agreement with observational abundances for the two observed isomers in Sgr B2(M), within an order of magnitude, occurs during the warm-up stage between
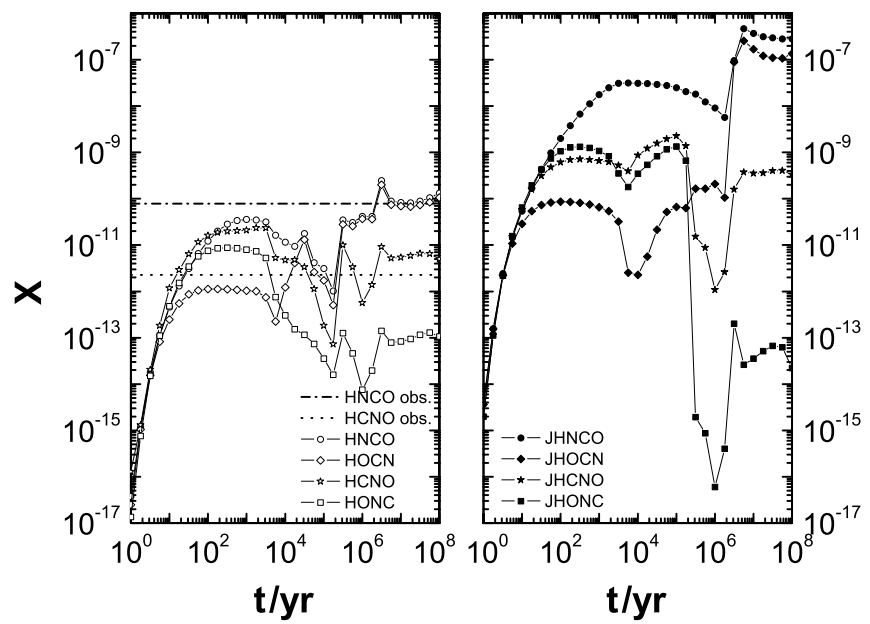

Figure 5. Calculated fractional abundances with respect to $n_{\mathrm{H}_{2}}$ for CHNO isomers in the gas (left panel, open symbols) and on dust particles (right panel, solid symbols) as functions of time in the lukewarm model. The horizontal lines represent the observed $\mathrm{HNCO}$ and $\mathrm{HCNO}$ fractional abundances toward L1527 (Marcelino et al. 2010).

1.6 and $1.8 \times 10^{5} \mathrm{yr}$ and during the $200 \mathrm{~K}$ stage between 7.1 and $12.1 \times 10^{5} \mathrm{yr}$. During these two time periods, the calculated fulminic acid (HCNO) abundance is also in good agreement with the upper limit set by observers (Marcelino et al. 2010). In other words, best agreement occurs both before and during the hotcore stage, with the former occurring between 27 and 40 K. Since the rotational excitation temperature of the detected isomers is $100 \mathrm{~K}$ and Sgr B2(M) is a hot core, the latter time interval is the more reasonable one, although it occurs at somewhat longer times than previously estimated chemical lifetimes for hot cores of $10^{4}-10^{5} \mathrm{yr}$ (Charnley et al. 1992). During this interval, the predicted abundances of isofulminic acid (HONC) remain much lower than the abundances of the lower energy isomers. We also note that the best agreement with observation for $\mathrm{HNCO}$ and HOCN in Sgr B2(N) occurs much closer to the end of the warm-up period, where the computed fractional abundances are at their peak. Finally, the non-detection of HCNO was not really explained in terms of purely gas-phase models and our previous gas-grain results (Marcelino et al. 2010); its low predicted abundance during the hot-core stage with our current model, well below that of HOCN after $2 \times 10^{4} \mathrm{yr}$, is in good agreement with observation. Figure 3 shows the HOCN/HNCO and $\mathrm{HCNO} / \mathrm{HNCO}$ ratios as functions of time, indicating how strongly $\mathrm{HCNO} / \mathrm{HNCO}$ diminishes after $10^{4} \mathrm{yr}$. It is likely that $\mathrm{HCNO}$ is destroyed more rapidly than $\mathrm{HOCN}$ because its destruction reaction with atomic oxygen at $200 \mathrm{~K}$ is rather rapid whereas that of $\mathrm{HOCN}$ is not (see Reaction (10) and the following discussion). The situation is less clear-cut at lower temperatures. An additional reason is the fact that the reaction between $\mathrm{H}$ and HOCN is no longer included in our model.

To simulate the cooler envelopes of the hot cores, we utilize a warm-up model with a peak temperature of $50 \mathrm{~K}$. According to Brünken et al. (2010), the rotational excitation temperature of the detected isomers around Sgr B2(M) and $(\mathrm{N})$ is $12-14 \mathrm{~K}$. We also use this model for $\mathrm{Sgr} \mathrm{B} 2(\mathrm{OH})$, which is not obviously along the line of sight to a hot core, but where the rotational temperature of HOCN is $20 \mathrm{~K}$ and the observed abundances are perhaps better constrained (Brünken et al. 2009a). Figure 4 shows calculated results of this model for both gaseous and surface abundances of the four isomers along with observational abundances of HNCO and HOCN toward Sgr B2(OH) based 

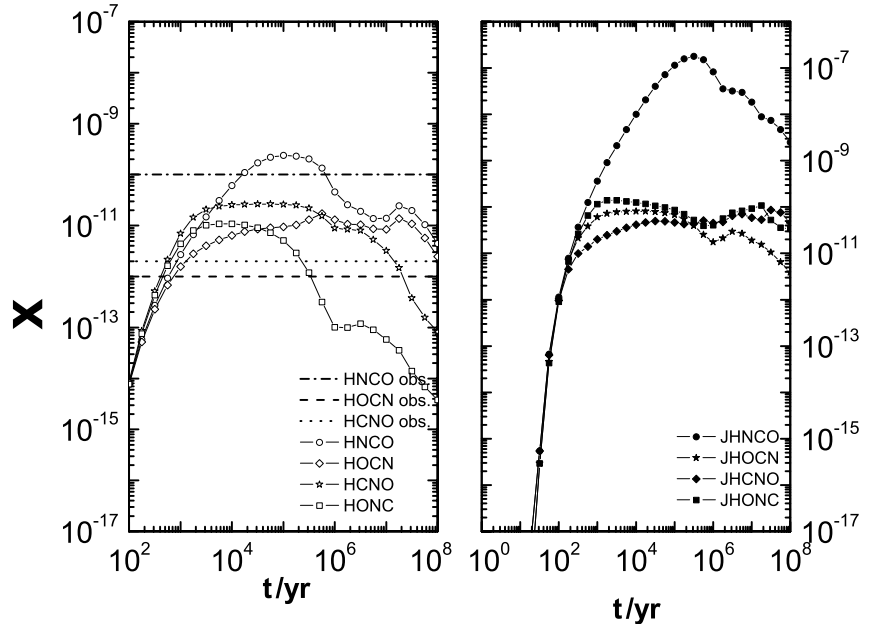

Figure 6. Calculated fractional abundances with respect to $n_{\mathrm{H}_{2}}$ for $\mathrm{CHNO}$ isomers in the gas (left panel, open symbols) and on dust particles (right panel, solid symbols) as functions of time in the cold-core model. The horizontal lines represent the observed $\mathrm{HNCO}, \mathrm{HCNO}$, and $\mathrm{HOCN}$ fractional abundances toward L1544 (Marcelino et al. 2010).

on the work of Churchwell et al. (1986), Turner (1991), and Brünken et al. (2009a). The gas-phase results are somewhat similar to those obtained for the hot-core model except that here the peak values of the gas-phase isomers are smaller and the decrease at times following the peak abundance less severe. Moreover, the depletion of surface species after the warm up is not as significant as that in the hot core, presumably because sublimation remains incomplete at $50 \mathrm{~K}$. Here, excellent agreement for $\mathrm{HNCO}$ occurs in one continuous period of time between $2 \times 10^{5}$ and $1.1 \times 10^{6} \mathrm{yr}$, while agreement with HOCN is better at two distinct times: before $2.4 \times 10^{5} \mathrm{yr}$, during the cold and warm-up stages, and between $7.1 \times 10^{5}$ and $1.4 \times 10^{7} \mathrm{yr}$, during the $50 \mathrm{~K}$ stage. In between these two intervals, the predicted abundance for HOCN is too large. For this comparison, the temperatures of best agreement during the warm-up stage make more physical sense. Unlike HNCO and HOCN, the calculated fractional abundance of HCNO is not very sensitive to time and hovers about $10^{-11}$ to $10^{-12}$, possibly explaining its non-abundance although the computed value still exceeds the upper limit, while the predicted abundance of HONC is lower.

Figure 5 shows modeling results for the lukewarm model along with observed abundances for $\mathrm{HNCO}$ and $\mathrm{HCNO}$ in the lukewarm corino L1527 (Marcelino et al. 2010). The abundance of HCNO is very similar to that of HOCN (Marcelino et al. 2010). Unlike the other two warm-up models, the temperature increase does not introduce a dramatic increase in the gas-phase fractional abundances of any isomers, nor does the subsequent $T=30 \mathrm{~K}$ period cause an apparent decrease of any isomers except for surface HONC. After a short time of $100 \mathrm{yr}$, both calculated $\mathrm{HNCO}$ and $\mathrm{HCNO}$ fractional abundances in the gas phase remain in very good (a factor of 3 to order of magnitude) agreement with observed abundances except for a brief dip at around $10^{5} \mathrm{yr}$. The calculated gaseous HOCN abundance is very low until $1 \times 10^{4} \mathrm{yr}$, after which it starts increasing, reaching a high value of $1 \times 10^{-11}$, and remaining abundant afterward except for a brief dip. The HONC isomer has a similar abundance to HCNO until $1 \times 10^{4} \mathrm{yr}$, after which it decreases and remains below $10^{-12}$. A reasonable time for agreement with the observed abundances of HNCO, HOCN, and HCNO occurs somewhat after the warm-up period; at $10^{6} \mathrm{yr}$, for example, the calculated abundances of HNCO and HCNO are in very good agreement with observations, while the abundance of HOCN is perhaps an order of magnitude too large. Agreement at earlier times would contradict the idea of a lukewarm corino.

Calculated gas-phase and surface abundances for the four isomers are shown for our cold-core model in Figure 6. This model clearly pertains to the cold cores studied by Marcelino et al. (2009) and Marcelino et al. (2010); the observed results shown in the figure for $\mathrm{HNCO}, \mathrm{HCNO}$, and $\mathrm{HOCN}$ are from L1544 since the density of this cold source is only slightly higher than used in the calculation. After $1 \times 10^{5} \mathrm{yr}$, the results of the cold-core model are quite distinct from the warm-up models since there is no warm up and much of the material remains on the ice. For HNCO, the gas-phase abundance reaches the observed value of $\approx 10^{-10}$ at a time of $10^{4} \mathrm{yr}$ and then oscillates around it, always within an order of magnitude of the observed value. The isomer HCNO is lower by a factor of about an order of magnitude from $\approx 5 \times 10^{4}$ to $5 \times 10^{5} \mathrm{yr}$ and is about an order of magnitude above its observed value during this time. The isomer HOCN is calculated to lie below HCNO in abundance until $10^{6} \mathrm{yr}$, and also lies at most an order of magnitude above the observed value. There is no sharply defined period of best agreement, although the standard early time of $\approx 10^{5} \mathrm{yr}$ is as reasonable a time as any. The calculated abundance for the metastable isomer HONC is here predicted to be comparable with the other metastable isomers until early time, so that it might be possible to detect HONC in cold sources (Mladenović et al. 2009).

\section{DISCUSSION}

We have used the OSU gas-grain chemical network with the addition of suitable reactions to model the abundances of four of the CHNO isomers-HNCO (isocyanic acid), HOCN (cyanic acid), HCNO (fulminic acid), and HONC (isofulminic acid). Models have been created for four different environments: a cold core, in which a constant temperature of $10 \mathrm{~K}$ is assumed, and three types of sources in which a gradual quiescent warm up occurs due to the formation of a star. In the hot-core model, the warm up occurs to a temperature of $200 \mathrm{~K}$ whereas in the model for the area surrounding a hot core, the warm up produces an asymptotic temperature of $50 \mathrm{~K}$. In addition to these two models, which pertain to medium-to-high-mass star formation, we use a warm-up model of a lukewarm corino such as L1527 (Sakai et al. 2008), surrounding a low-mass protostar, in which the temperature only rises to $30 \mathrm{~K}$. Raising the hotcore temperature to $300 \mathrm{~K}$ results in little change for the $\mathrm{CHNO}$ isomeric abundances at the end of the warm-up period and for some time thereafter.

The four CHNO isomers are produced by a combination of surface chemistry and gas-phase chemistry, which are often difficult to separate, as found previously for HNCO by Tideswell et al. (2010). For example, in the hot-core model, HNCO is initially formed mainly by surface recombination of $\mathrm{JH}$ and JNCO followed by non-thermal desorption into the gas, but at $5.6 \times 10^{3} \mathrm{yr}$, dissociative recombination of $\mathrm{H}_{2} \mathrm{NCO}^{+}$takes over, while during the warm-up stage, evaporation of JHNCO dominates. For the cold-core model, gas-phase reactions play a more prominent role, and one gas-phase reaction (Reaction (4)) is particularly important for the formation of $\mathrm{HCNO}$ (Marcelino et al. 2009, 2010). Now that the ion $\mathrm{H}_{2} \mathrm{NCO}^{+}$has been detected in the laboratory (Lattanzi et al. 2010), it might be possible to detect it in space although its predicted abundance is rather low. 
In all environments observed to date, the lowest energy species (HNCO) is typically the most abundant, with the other observed or unobserved isomers calculated to be lower in abundance by a factor of $\approx 10-100$. This calculated factor is, however, often somewhat smaller than the typical observational factor of $\approx 100$. We reproduced the observational factor for larger time intervals in preliminary calculations until it was found by quantum chemical techniques that HNCO reacts with the relatively abundant atomic carbon, thus reducing its abundance with respect to the other isomers. Indeed, we are forced to assume the rate coefficient of this reaction to be somewhat less than the typical gas-kinetic value to produce enough HNCO to maintain a factor of at least 10.

Concerning the higher energy isomers, if one looks at the observed $\mathrm{HCNO} / \mathrm{HOCN}$ ratio, this ratio, around unity for cold sources, becomes considerably smaller for warm sources; indeed, $\mathrm{HCNO}$ is not detected in the warm sources at all. Our calculations show a reasonable period of time in which cold cores have nearly equal abundances for these two metastable isomers; for the hottest sources, during the hot-core period, the abundance of HOCN is predicted to be considerably higher than that of HCNO.

The high abundance of $\mathrm{HNCO}$ compared with the other isomers is analogous to the case of $\mathrm{HC}_{3} \mathrm{~N}$ and its higher energy isomers (Osamura et al. 1999). The $\mathrm{HCNO} / \mathrm{HOCN}$ ratio, on the other hand, is similar to the $\mathrm{HNC} / \mathrm{HCN}$ ratio, in which the relative abundance of the higher energy isomer (HNC) becomes smaller with respect to $\mathrm{HCN}$ as the temperature increases (Herbst et al. 2000).

In general, our models are able to reproduce adequately both the abundance of the dominant isomer $\mathrm{HNCO}$ and the abundance or upper limit of the minor isomers, $\mathrm{HCNO}$ or $\mathrm{HOCN}$, for significant periods of time. This agreement is superior to what we reported in an earlier paper (Marcelino et al. 2010), in which reactions involving atomic $\mathrm{H}$ (see Reactions (13) and (14)) reduced the abundance of HOCN considerably compared with HCNO. Here we neglected these reactions, based initially on the assumption that they possess activation energy, in analogy with Reaction (15). Preliminary calculations have indeed led to a barrier for $\mathrm{H}+\mathrm{HOCN}$. For the warm-up models of a hot core and its cooler envelope, there can be two periods of best agreement: one during the warm-up period and one during the constant hightemperature phase. For the lukewarm corino case, reasonable agreement occurs over a long period, but makes more physical sense after the warm up. Finally, for the cold core, the agreement is reasonable during the early time period usually associated with best overall agreement for gas-phase species. The highest energy isomer of the four studied, HONC (isofulminic acid), has not yet been detected in the interstellar medium but according to our calculations here, it should be detectable, albeit weakly. The use of a gas-grain model for the environments studied is supported by the recent study of Rodríguez-Fernández et al. (2010) in which shock enhancement of HNCO is detected in the L1157 molecular outflow. Indeed, if all of the HNCO calculated to be on the grain surface at its peak abundance could be blown off the grains in L1157, the gas-phase HNCO abundance would reach a value close to $10^{-7}$, which is in good agreement with the observed value of Rodríguez-Fernández et al. (2010).

Finally, the CHNS isomers present another interesting case of how astronomical environments lead to the production and destruction of differing isomers. The lowest energy formHNCS-is a well-known interstellar molecule, and the metastable isomer HSCN has just been detected toward Sgr
B2(N) (Halfen et al. 2009) based on recent laboratory evidence (Brünken et al. 2009b). Inclusion of these and higher energy isomers into our gas-grain network has been undertaken (Adande et al. 2010).

We thank S. Brünken for a careful reading of the manuscript. E.H. acknowledges the support of the National Science Foundation for his astrochemistry program through grant AST0702876, and his program in chemical kinetics through the Center for the Chemistry of the Universe. He also acknowledges support from NASA NAI for studies in the evolution of pre-planetary matter. E.R. acknowledges support from PCMI, a national Program on Interstellar Molecules of CNRS-INSU.

\section{REFERENCES}

Adande, G. R., Halfen, D. T., Liurys, L. M., Quan, D., \& Herbst, E. 2010, ApJ, in press

Becke, A. D. 1993, J. Chem. Phys., 98, 5648

Brown, R. 1981, ApJ, 248, L119

Brünken, S., Belloche, A., Martín, S., Verheyen, L., \& Menten, K. M. 2010, A\&A, 516, A109

Brünken, S., Gottlieb, C. A., McCarthy, M. C., \& Thaddeus, P. 2009a, ApJ, 697, 880

Brünken, S., Yu, Z., Gottlieb, C. A., McCarthy, M. C., \& Thaddeus, P. 2009b, ApJ, 706, 1588

Charnley, S. B., Tielens, A. G. G. M., \& Millar, T. J. 1992, ApJ, 399, L71

Churchwell, E., Woon, D., Myers, P. C., \& Myers, R. V. 1986, ApJ, 305, 405

Dunning, T. H. 1989, J. Chem. Phys., 90, 1007

Eschenko, G., Koecher, T., Kerst, C., \& Temps, F. 2002, Chem. Phys. Lett., 356, 181

Feng, W., \& Hershberger, J. F. 2007, J. Phys. Chem., A111, 10654

Fikri, M., Meyer, S., Roggenbuck, J., \& Temps, F. 2001, Faraday Discuss., 119, 223

Frisch, M. J., et al. 2004, GUASSIAN 03 Revision D.02 (Wallingford, CT: Gaussian Inc.)

Garrod, R., \& Herbst, E. 2006, A\&A, 457, 927

Garrod, R., Wakelam, V., \& Herbst, E. 2007, A\&A, 467, 1103

Glarborg, P., Alzueta, M. U., Dam-Johansen, K., \& Miller, J. A. 1998, Combust. Flame, 115, 1

Halfen, D. T., Ziurys, L. M., Brünken, S., Gottlieb, C. A., McCarthy, M. C., \& Thaddeus, P. 2009, ApJ, 702, L124

Hasegawa, T. I., \& Herbst, E. 1993, MNRAS, 263, 589

Hasegawa, T. I., Herbst, E., \& Leung, C. M. 1992, ApJS, 83, 167

Hassel, G. E., Herbst, E., \& Garrod, R. T. 2008, ApJ, 681, 1385

Herbst, E., \& Millar, T. J. 2008, in Low Temperatures and Cold Molecules, ed. I. W. M. Smith (London: Imperial College Press), 1

Herbst, E., Terzieva, R., \& Talbi, D. 2000, MNRAS, 311, 869

Iglesias, E. 1977, ApJ, 218, 697

Jansen, D. J., Spaans, M., Hogerheijde, M. R., \& van Dishoeck, E. F. 1995, A\&A, 303, 541

Lattanzi, V., Gottlieb, C. A., Thaddeus, P., McCarthy, M. C., \& Thorwirth, S. 2010, Int. Symp. Mol. Spec., 65, FC15 (Columbus, OH: Ohio State Univ.), (http://spectroscopy.mps.ohio-state.edu/symposium/)

Lee, C., Yang, W., \& Parr, R. G. 1988, Phys. Rev. B, 37, 785

Liu, S.-Y., \& Snyder, L. E. 1999, ApJ, 523, 683

Marcelino, N., Brünken, S., Cernicharo, J., Quan, D., Roueff, E., Herbst, E., \& Thaddeus, P. 2010, A\&A, 516, A105

Marcelino, N., Cernicharo, J., Tercero, B., \& Roueff, E. 2009, ApJ, 690, L27

Martín, S., Martín-Pintado, J., \& Viti, S. 2009, ApJ, 706, 1323

Martín, S., Requena-Torres, M. A., Martín-Pintado, J., \& Mauersberger, R. 2008, ApJ, 678, 245

Menten, K. M. 2004, in The Dense Interstellar Medium in Galaxies, ed. S. Pfalzner, C. Kramer, C. Staubmeir, \& A. Heithausen (New York: Springer), 69

Miller, J. A., Durant, J. L., \& Glarborg, P. 1998, Combust. Flame, 135, 357

Miller, J. A., Klippenstein, S. J., \& Glarborg, P. 2003, Proc. Comb. Inst., 27, 234

Mladenović, M., Lewerenz, M., McCarthy, M. C., \& Thaddeus, P. 2009, J. Chem. Phys., 131, 174308

Osamura, Y., Fukuzawa, K., Terzieva, R., \& Herbst, E. 1999, ApJ, 519, 697

Pople, J. A., Head-Gordon, M., \& Raghavachari, K. 1987, J. Chem. Phys., 87, 5968 
Rodríguez-Fernández, N. J., Tafalla, M., Gueth, F., \& Bachiller, R. 2010, A\&A, 516, A98

Roggenbuck, J., \& Temps, F. 1998, Chem. Phys. Lett., 285, 422

Ruffle, D. P., \& Herbst, E. 2001, MNRAS, 322, 770

Sakai, N., Sakai, T., Hirota, T., \& Yamamoto, S. 2008, ApJ, 672, 371

Schuurman, M. S., Muir, S. R., Allen, W. D., \& Schaefer, H. F., III. 2004, J. Chem. Phys., 120, 11586

Snyder, L. E., \& Buhl, D. 1972, ApJ, 177, 619
Talbi, D., Ellinger, Y., \& Herbst, E. 1996, A\&A, 314, 688

Tideswell, D. M., Fuller, G. A., Millar, T. J., \& Markwick, A. J. 2010, A\&A, 510, A85

Turner, B. E. 1991, ApJS, 76, 617

Turner, B. E., Terzieva, R., \& Herbst, E. 1999, ApJ, 518, 699

Viti, S., Collings, M. P., Dever, J. W., McCoustra, M. R. S., \& Williams, D. A. 2004, MNRAS, 354, 1141

Zhang, W., Du, B., \& Feng, C. 2004, J. Mol. Struct. (Theochem), 679, 121 